\title{
SUBNORMALITY IN SOLUBLE MINIMAX GROUPS
}

Dedicated to the memory of Hanna Neumann

$$
\text { D. J. McCAUGHAN }
$$

(Received 15 August 1972)

Communicated by M. F. Newman

\section{Introduction}

A subgroup $H$ of a group $G$ is said to be subnormal in $G$ if there is a finite chain of subgroups, each normal in its successor, connecting $H$ to $G$. If such chains exist there is one of minimal length; the number of strict inclusions in this chain is called the subnormal index, or defect, of $H$ in $G$. The rather large class of groups which have an upper bound for the subnormal indices of their subnormal subgroups has been investigated to some extent, mainly with a restriction to soluble groups - for instance, in [10], McDougall considered soluble p-groups in this class. Robinson, in [14], restricted his attention to wreath products of nilpotent groups but extended his investigations to the strictly larger class of groups in which the intersection of any family of subnormal subgroups is a subnormal subgroup. These groups are said to have the subnormal intersection property.

In [17] Roseblade studied groups in which every subgroup is subnormal, and proved that if a group of this type has bounded subnormal indices then it is nilpotent, and its class is bounded by a function of the bound for the subnormal indices. In [8] McDougall and the present author obtained some information on the structure of metanilpotent groups with bounded subnormal indices; the corresponding results for groups with the subnormal intersection property were less satisfactory. From a consideration of such attempts to describe the structure of groups which have some restrictions on their subnormal subgroups, it is clear that the treatment of groups with bounds on their subnormal indices is relatively easy when compared with that of groups with the subnormal intersection property. It is therefore of some interest to have at least a partial answer to the question: for which classes of groups do these two restrictions coincide? The results of [14] show that for wreath products of nilpotent groups they do not. But, in [12], Robinson characterised finitely generated soluble groups with the subnormal 
intersection property, proving in the process that such groups have bounded subnormal indices; thus at least for finitely generated soluble groups the two restrictions are equivalent.

In section 4 of this paper we show that a similar statement holds for the class of soluble minimax groups, that is, soluble groups which have a subnormal series of finite length in which each factor satisfies the minimal or the maximal condition on subgroups. Our fundamental result is theorem 3.2, the proof of which occupies the bulk of section 3. Section 5 is devoted to the construction of a particular group which has the subnormal intersection property but unbounded subnormal indices. Although this group is not a minimax group, it lies in the related class of soluble groups of finite reduced rank, that is, soluble groups in which every finitely generated subgroup can be generated by a fixed number of elements.

\section{Notation and preliminary results}

In this section we aim to summarise, for convenience, some basic facts and terminology which will be used implicitly in later sections.

SUBNoRmal SUbGroups. Our treatment of subnormal subgroups leans heavily on the idea of standard series of a subgroup. If $H$ is a subgroup of a group $G$, the standard series of $H$ in $G$ may be defined by: $H^{G .0}=G, H^{G, i}$ is the normal closure of $H$ in $H^{G, i-1}$ if $i$ is a positive integer. It is easy to show that $H$ is subnormal in $G$ if and only if for some integer $n, H=H^{G, n}$; the least such $n$ is of course the subnormal index of $H$ in $G$, denoted throughout by $s(G: H)$. As is well known, for each non-negative integer $r$, the $r$-th term of the standard series of $H$ in $G$ is given by $H^{G . r}=H \gamma G H^{r}$, where $\gamma G H^{r}$ is defined inductively: $\gamma G H^{0}=G$ and $\gamma G H^{r+1}$ $=\left[\gamma G H^{r}, H\right]$. Thus if $H$ is subnormal in $G$ the subnormal index $s(G: H)$ is the least integer $r$ for which $\gamma G H^{r} \leqq H$.

We are conzeraed with two classes of groups, the first comprising those groups which have the subnormal intersection property, the second those which have bounds on the subnormal indices of their subnormal subgroups. The groups in these classes can be usefully characterised by the behaviour of the standard series of an arbitrary subgroup, the characterisation showing incidentally that the second class is indeed a subclass of the first.

Lemma 2.1. (lemma 2 of [12]) (i) A group $G$ has the subnormal intersection property if and only if for each subgroup $H$ of $G$ there is an integer $n$ depending on $H$ such that $H^{G \cdot n}=H^{G \cdot n+1}$, that is, the standard series of each subgroup in $G$ becomes stationary after finitely many steps.

(ii) $A$ group $G$ has a bound $k$ on the subnormal indices of its subnormal subgroups if and only if for each subgroup $H$ of $G, H^{G, k}=H^{G \cdot k+1}$. 
It is worth noting that if a group $G$ lies in either of these two classes then any homomorphic image or subnormal subgroup of $G$ will lie in the same class. Moreover, in [12] and [14], Robinson provides examples of groups which have the subnormal intersection property but unbounded subnormal indices - for instance the group $C_{p} w r C_{p \infty}$, where $p$ is any prime.

Commutator Resulrs. We include for easy reference our two main tools for the manipulation of commutator subgroups. The first of these is a weak form of the "three subgroup lemma" of Hall.

Lemma 2.2. ([3], Theorem 2.3.) If $H, K$ and $L$ are normal subgroups of $a$ group, then $[H, K, L] \leqq[K, L, H][L, H, K]$.

Although our second tool is also essentially due to Hall (Theorem 2 of [4]) it is convenient to give a slightly sharper version of his result, which may be established by a simple inductive argument which we omit.

LEMMA 2.3. If $H$ is a normal subgroup of $G$ then for each positive integer $k$, $\left[H, \gamma_{k} G\right] \leqq \gamma H G^{k}$, where $\gamma_{k} G$ denotes the $k$-th term of the lower central series of $G$.

RADICABILITY. If $\pi$ is any set of primes, we say that a group is quasi- $\pi$-radicable if it can be generated by the $k$-th powers of its elements for each $\pi$-number $k$, that is, for each positive integer $k$ all of whose prime divisors lie in $\pi$. We say that the group is $\pi$-radicable if every element of the group is a $k$-th power for each $\pi$ number $k$. It is not difficult to see that in an arbitrary group $G$ the subgroup generated by a family of quasi- $\pi$-radicable subgroups is itself quasi- $\pi$-radicable, so that $G$ has a unique maximal quasi- $\pi$-radicable subgroup. This subgroup is fully invariant in $G$ since quasi- $\pi$-radicability is inherited by homomorphic images. If the maximal quasi- $\pi$-radicable subgroup of $G$ is trivial, $G$ is said to be $\pi$-reduced; the factor group of any group with respect to its maximal quasi $\pi$ radicable subgroup will be $\pi$-reduced. A well-known result of Cernikov [2] implies that any quasi- $\pi$-radicable $Z A$-group is actually $\pi$-radicable, although $\pi$-radicability is, in general, the stronger property. For the most part we will deal only with the case where $\pi$ contains but one prime $p$ : we will use such terms as $p$ radicable and $p$ reduced.

Finiteness Conditions. An abelian group $A$ is said to have finite reduced rank if there is an upper bound for the values of the 0 -rank and the $p$-rank of $A$ as $p$ ranges over the set of all primes. A soluble group of finite reduced rank is a group with a subnormal series of finite length in which the factors are abelian groups of finite reduced rank. A soluble minimax group is one with a subnormal series of finite length in which the factors are abelian groups satisfying the minimal or maximal condition on subgroups. It is well known that the class of soluble groups of finite reduced rank properly contains the class of soluble minimax 
groups. (See chapter 6 of [16].) As far as these classes are concerned, beyond the obvious remark that both are closed under the operations of forming subgroups and homomorphic images, we will require only the following result; the proof is elementary and is omitted.

LEMMA 2.4. Let $G$ be a soluble group of finite reduced rank. If $\left\{H_{i}: i \geqq 0\right\}$ is an ascending chain of normal subgroups of $G$ then all but finitely many of the factors $\left\{H_{i+1} / H_{i}: i \geqq 0\right\}$ are periodic.

This lemma will in fact be used in the context of soluble minimax groups: for further information on these we refer the reader to [1] and [15].

\section{The basic theorem}

To begin this section we introduce a concept which does not appear to have been studied elsewhere.

Definition 3.1. Let $G$ be a group and $N$ a normal subgroup of $G$. We denote by $\Sigma(G: N)$ the family of subnormal subgroups $S$ of $G$ such that $S N=G$. We put $\sigma(G: N)=\cap\{S: S \in \Sigma(G: N)\}$.

It is easy to see that $\sigma(G: N)$ is a normal subgroup of $G$; indeed if $N$ is a characteristic subgroup of $G$ then $\sigma(G: N)$ will also be characteristic in $G$.

If $N$ is a normal subgroup of $G$ and $N \leqq M \leqq G$, then for any $S$ in $\Sigma(G: N)$, $(S \cap M) N=S N \cap M=M$, and so $S \cap M \in \Sigma(M: N)$, showing that $\sigma(M: N)$ $\leqq M \cap \sigma(G: N)$.

Our fundamental theorem, in which this concept plays a part, follows a familiar pattern (compare theorem 5.4 of [10] or Lemma 4 of [12] in deducing the nilpotency of a group from conditions which partly involve subnormal subgroups.

THEOREM 3.2. Let $G$ be a group with a nilpotent normal subgroup $N$ such that $G / N$ is a finite nilpotent group. If $\sigma(G: N)=1$ and $G$ has the subnormal intersection property then $G$ is nilpotent.

Proof. In view of the second remark after defizition 3.1, it is easily seen that the hypotheses of the theorem apply to any normal subgroup of $G$ containing $N$. By Fitting's theorem we may therefore assume, without loss of generality, that $G / N$ is a finite $p$ group for some prime $p$.

Let $P$ denote the maximal $p$-radicable subgroup of $N$. Let us define a chain $\left\{P_{i}: i \geqq 0\right\}$ of normal subgroups of $G$ as follows: $P_{0}=1 ; P_{i} / P_{i-1}$ is the maximal $p$-radicable subgroup of $\zeta_{1}\left(N / P_{i-1}\right)$ (the centre of $\left.N / P_{i-1}\right)$, for $i \geqq 1$. By lemma 3.6 of [8], $P_{i}$ is precisely the maximal $p$ radicable subgroup of $\zeta_{i}(N)$, for each $i(0 \leqq i \leqq m)$ and $P_{m}=P_{m+1}=P$, where $m$ is the nilpotent class of $N$.

If, for $0<i \leqq m$, we denote by $C_{i}$ the centralizer in $G$ of the group $P_{i} / P_{i-1}$, our last statement implies that $N \leqq C_{i}$. Thus the action of $G$ on $P_{i} / P_{i-1}$ is es- 
sentially that of the finite $p$ group $G / C_{i}$, and we can now apply lemma 3 of [7]. We deduce that for each $i, P_{i}$ is the product of two subgroups $K_{i}$ and $L_{i}$ with the following properties

(i) $P_{i-1} \leqq K_{i} \cap L_{i}$,

(ii) $\left[L_{i}, G\right] \leqq P_{i-1}$,

(iii) $K_{i}=\left[K_{i}, G\right] P_{i-1}=\left[P_{i}, G\right] P_{i-1}$.

These equations hold for $0<i \leqq m$. If we put $K_{0}=L_{0}=1$, it is easy to see from (iii) that $\left[K_{i}, G\right] \geqq\left[K_{i-1}, G\right]$ and that (iii) can then be extended to

(iv) $K_{i}=\left[K_{i}, G\right] L_{i-1}=L_{i-1} \gamma K_{,} G^{r}$

for any integer $r \geqq 0$.

Finally, the centrality of $P_{i} / P_{i-1}$ in $N / P_{i-1}$ implies that for any $S$ in $\Sigma(G: N)$, we have, substituting $S N$ for $G$ in (iv), that

(v) $K_{i}=\left[K_{i}, S\right] L_{i-1}=L_{i-1} \gamma K_{i} S^{r}$

for any integer $r \geqq 0$.

Since we know from Theorem $A$ of [7] that $G / P$ is nilpotent, our objective is to prove that for some integer $k, \gamma P G^{k}=1$. We accomplish this by showing that for each $i$ with $0<i \leqq m, K_{i}=P_{i-1}$, that is, $P_{i}=L_{i}$ (which will show that in fact $\gamma P G^{m}=1$ ).

Suppose that this statement is false, and let $K_{t}$ be the first member of the chain $\left\{K_{i}: i>0\right\}$ which violates it. Then $K_{t}>P_{t-1}$. Firstly we note that if $S$ is an arbitrary subgroup in $\Sigma(G: N), \gamma K_{1} S^{r}=K_{1}$, for any $r \geqq 0$, by (v) above. From the subnormality of $S$ it then follows that $K_{1} \leqq S$ and thus that $K_{1} \leqq \sigma(G: N)=1$. This shows that $t>1$.

The minimality of $t$ implies that for any $i$ with $0<i<t, P_{i}=L_{i}$ and $\left[P_{i}, G\right] \leqq P_{i-1}$. An obvious inductive argument yields

$$
\gamma P_{t-1} G^{t-1}=\gamma L_{t-1} G^{t-1}=1 .
$$

Since $L_{t-1}$ is normal in $G$, we may apply lemma 2.3 to deduce that

$$
\left[L_{t-1}, \gamma_{t-1} G\right]=1 \text {. }
$$

Now we proceed to establish by induction on $i$ that for $0<i \leqq m$

$$
\left[P_{i} \cap \gamma_{t-1} G, K_{t}\right]=1 .
$$

Consider the subgroup $P_{t-1} \cap \gamma_{t-1} G=L_{t-1} \cap \gamma_{t-1} G$. We can write

$$
K_{t}=L_{t-1} \gamma K_{t} G^{r}
$$

for any non-negative integer $r$, by (iv). Thus

$$
\left[P_{t-1} \cap \gamma_{t-1} G, K_{t}\right]=\left[L_{t-1} \cap \gamma_{t-1} G, L_{t-1} \gamma K_{t} G^{t-1}\right] .
$$

Applying (b) twice, we see that 


$$
\left[P_{t-1} \cap \gamma_{t-1} G, K_{t}\right]=1,
$$

so that (c) holds for $0<i \leqq t-1$, at least.

Suppose now that (c) holds with $i=j<m$. We show first of all that

$$
\left[K_{j+1} \cap \gamma_{t-1} G, K_{t}\right]=1 \text {. }
$$

Let us denote by $M_{j+1}$ the commutator which appears in (d). If $S$ is any subgroup in $\Sigma(G: N)$, say of defect $s$ in $G$, we may choose an integer $r \geqq s+t$ and write, using (v),

$$
M_{j+1}=\left[K_{j+1} \cap \gamma_{t-1} G, K_{t}\right]=\left[\left(L_{j} \gamma K_{j+1} S^{r}\right) \cap \gamma_{t-1} G, K_{t}\right] .
$$

By the choice of $r$,

$$
\gamma K_{j+1} S^{r} \leqq \gamma_{t-1} G,
$$

and so we have, by the modular law,

$$
M_{j+1}=\left[\left(L_{j} \cap \gamma_{t-1} G\right) \gamma K_{j+1} S^{r}, K_{t}\right]
$$

But by our induction hypothesis,

so we have

$$
\left[L_{j} \cap \gamma_{t-1} G, K_{t}\right]=1
$$

$$
M_{j+1}=\left[\gamma K_{j+1} S^{r}, K_{t}\right]
$$

Now, using (v) again, we may write

$$
M_{j+1}=\left[\gamma K_{j+1} S^{r}, L_{t-1} \gamma K_{t} S^{r}\right]
$$

which, recalling the choice of $r$ and (b) above, becomes

$$
M_{j+1}=\left[\gamma K_{j+1} S^{r}, \gamma K_{t} S^{\prime}\right] \text {. }
$$

Since $r$ exceeds the defect $s$ of $S$ in $G$, we have shown that $M_{j+1} \leqq S$. It follows that, since $S$ was an arbitrary member of $\Sigma(G: N)$,

$$
M_{j+1}=\left[K_{j+1} \cap \gamma_{t-1} G, K_{t}\right] \leqq \sigma(G: N)=1,
$$

proving (d).

Let us denote by $R_{j+1}$ the commutator

$$
\left[P_{j+1} \cap \gamma_{t-1} G, K_{t}\right]=\left[K_{t}, P_{j+1} \cap \gamma_{t-1} G\right] \text {. }
$$

Using (iv) we may write

$$
R_{j+1}=\left[L_{t-1}\left[K_{t}, G\right], P_{j+1} \cap \gamma_{t-1} G\right]
$$

which, in view of (b), becomes

$$
R_{j+1}=\left[K_{t}, G, P_{j+1} \cap \gamma_{t-1} G\right]
$$


Applying the three subgroup lemma 2.2 we deduce that

$$
R_{j+1} \leqq\left[G, P_{j+1} \cap \gamma_{t-1} G, K_{t}\right]\left[P_{j+1} \cap \gamma_{t-1} G, K_{t}, G\right]
$$

Now by (iii) we have

$$
\left[G, P_{j+1} \cap \gamma_{t-1} G, K_{t}\right] \leqq\left[K_{j+1} \cap \gamma_{t-1} G, K_{t}\right]
$$

and so by (d) above

$$
\left[G, P_{j+1} \cap \gamma_{t-1} G, K_{t}\right]=1
$$

It then follows that

$$
R_{j+1} \leqq\left[P_{j+1} \cap \gamma_{t-1} G, K_{t}, G\right]=\left[R_{j+1}, G\right] .
$$

But now

$$
\begin{aligned}
{\left[R_{j+1}, N\right] } & =\left[P_{j+1} \cap \gamma_{t-1} G, K_{t}, N\right] \\
& \leqq\left[K_{t}, N, P_{j+1} \cap \gamma_{t-1} G\right]\left[N, P_{j+1} \cap \gamma_{t-1} G, K_{t}\right]
\end{aligned}
$$

by the three subgroup lemma. The second of these commutators is trivial, by (e), hence

$$
\begin{aligned}
{\left[R_{j+1}, N\right] } & \leqq\left[K_{t}, N, P_{j+1} \cap \gamma_{t-1} G\right] \\
& \leqq\left[P_{t-1}, P_{j+1} \cap \gamma_{t-1} G\right]=\left[L_{t-1}, P_{j+1} \cap \gamma_{t-1} G\right],
\end{aligned}
$$

which is trivial, by (b) above.

Since $\left[R_{j+1}, N\right]=1$, it follows from (f) that for any subgroup $S$ in $\Sigma(G: N)$,

$$
R_{j+1} \leqq\left[R_{j+1}, G\right]=\left[R_{j+1}, N S\right]=\left[R_{j+1}, S\right]
$$

It is then clear that for any $r \geqq 0$,

$$
\gamma R_{j+1} S^{r}=R_{j+1}
$$

Since $S$ is subnormal in $G$, we must have $R_{j+1} \leqq S$, and so $R_{j+1} \leqq \sigma(G: N)=1$. This proves that

$$
\left[P_{j+1} \cap \gamma_{t-1} G, K_{t}\right]=1,
$$

showing that (c) holds for each $i$ with $0 \leqq i \leqq m$, as claimed.

Before proceeding with the proof of the theorem, we digress to prove an essential equality. Let $Y$ be any normal subgroup of $G$ which is contained in $N$, and denote by $Y_{(i)}$ the subgroup $\left[\gamma Y G^{i}, K_{t}\right]$, for each $i \geqq 0$. Then

$$
\gamma Y_{(0)} N^{t-2}=\gamma Y_{(i)} N^{t-2} \text { for each } i \geqq 0 .
$$

Since we know that $t>1$, these commutators are defined. We will establish (g) by showing that for $i \geqq 0$, 


$$
\gamma Y_{(i)} N^{t-2}=\gamma Y_{(i+1)} N^{t-2}
$$

Now, for each $i \geqq 0$,

$$
\begin{aligned}
Y_{(i)} & =\left[K_{t}, \gamma Y G^{i}\right] \\
& =\left[\left[K_{t}, G\right] L_{t-1}, \gamma Y G^{i}\right] \quad \text { by (iv). }
\end{aligned}
$$

From this we obtain

$$
Y_{(i)} \leqq\left[K_{t}, G, \gamma Y G^{i}\right]\left[L_{t-1}, G\right] .
$$

Using the three subgroup lemma 2.2 we then have

$$
Y_{(i)} \leqq\left[G, \gamma Y G^{i}, K_{t}\right]\left[\gamma Y G^{i}, K_{t}, G\right]\left[L_{t-1}, G\right],
$$

since all the subgroups involved are normal in $G$.

Now, since $Y \leqq N$,

$$
\left[\gamma Y G^{i}, K_{t}\right] \leqq P_{t-1}=L_{t-1} .
$$

It then follows that

$$
Y_{(i)} \leqq\left[\gamma Y G^{i+1}, K_{t}\right]\left[L_{t-1}, G\right] \text { or } Y_{(i)} \leqq Y_{(i+1)}\left[L_{t-1}, G\right] .
$$

Then it is clear that

$$
\gamma Y_{(i)} N^{t-2} \leqq\left(\gamma Y_{(i+1)} N^{t-2}\right) \gamma\left[L_{t-1}, G\right] N^{t-2}
$$

Since, by (a),

we must have

$$
\gamma L_{t-1} G^{t-1}=1
$$

$$
\gamma Y_{(i)} N^{t-2} \leqq \gamma Y_{(i+1)} N^{t-2}
$$

The reverse inclusion being trivial, we have established the desired equality, and with it (g).

Returning to the main stream of the proof, we now show by induction on $i$ that, for each $i$ with $0<i \leqq m$,

$$
\gamma\left[P_{i}, K_{t}\right] N^{t-2}=1 .
$$

From a consideration of equality (a) above, it is clear that (h) is valid for $0<i \leqq t-1$. Let $j$ be an integer with $t-1 \leqq j<m$, and suppose that

$$
\gamma\left[P_{j}, K_{t}\right] N^{t-2}=1
$$

We seek to show that

$$
\gamma\left[P_{j+1}, K_{t}\right] N^{t-2}=1,
$$

but since $\left[P_{j+1}, G\right] \leqq K_{j+1}$ by (iii), we see that, in view of $(\mathrm{g})$, we need only establish the equality 


$$
\gamma\left[K_{j+1}, K_{t}\right] N^{t-2}=1 .
$$

But by (iv) we may write

$$
\begin{aligned}
\gamma\left[K_{j+1}, K_{t}\right] N^{t-2} & =\gamma\left[L_{j} \gamma K_{j+1} G^{t}, K_{t}\right] N^{t-2} \\
& =\gamma\left[\gamma K_{j+1} G^{t}, K_{t}\right] N^{t-2} \gamma\left[L_{j}, K_{t}\right] N^{t-2} .
\end{aligned}
$$

By the induction hypothesis, this last commutator is trivial, so

$$
\begin{aligned}
\gamma\left[K_{j+1}, K_{t}\right] N^{t-2} & =\gamma\left[\gamma K_{j+1} G^{t}, K_{t}\right] N^{t-2} \\
& \leqq \gamma\left[P_{j+1} \cap \gamma_{t-1} G, K_{t}\right] N^{t-2}
\end{aligned}
$$

which is trivial, by (c). Hence

$$
\gamma\left[P_{j+1}, K_{t}\right] N^{t-2}=1,
$$

and the proof of $(\mathrm{h})$ is complete.

Taking $i=m$ in (h) yields

$$
\gamma\left[P_{m}, K_{t}\right] N^{t-2}=\gamma\left[P, K_{t}\right] N^{t-2}=1 .
$$

Since $G / P$ is nilpotent, by our previous remarks, there is a non-negative integer $r$ such that $\gamma N G^{r} \leqq P$. Then, recalling the notation of $(\mathrm{g})$ above, we have

$$
\left[\gamma N G^{r}, K_{t}\right]=N_{(r)} \leqq P_{(0)}=\left[P, K_{t}\right] .
$$

Since $\gamma P_{(0)} N^{t-2}=1$, we then have, by $(\mathrm{g})$, with $Y=N$,

$$
\gamma N_{(0)} N^{t-2}=\gamma N_{(r)} N^{t-2} \leqq \gamma P_{(0)} N^{t-2}=1 .
$$

In other words

hence

$$
1=\gamma\left[N, K_{t}\right] N^{t-2}=\gamma K_{t} N^{t-1},
$$

$$
K_{t} \leqq \zeta_{t-1}(N)
$$

Now $K_{t} / P_{t-1}$ is generated by homomorphic images of $P_{t} / P_{t-1}$ (see (iii)) and so is quasi-p-radicable. Then $K_{t}$ is a $p$-radicable subgroup of $\zeta_{t-1}(N)$, and

$$
K_{t} \leqq P_{t-1},
$$

contradicting our original assumption on $K_{t}$. By remarks at the beginning of the proof, this contradiction establishes that $\gamma P G^{m}=1$, and since $G / P$ is known to be nilpotent, we deduce that $G$ is nilpotent, completing the proof. theorem.

By using some results of [7] we can prove an interesting corollary to this

COROLlary 3.3. Let $\pi$ be a non-empty set of primes and $G$ a group with a $\pi$-torsion-free nilpotent normal subgroup $N$ such that $G / N$ is a nilpotent $\pi$-group. 
If $\sigma(G: N)=1$ and $G$ has the subnormal intersection property, then $G$ is nilpotent.

Proof. By Theorem $\mathrm{C}$ of [7], $G$ has a bound $k$ on its subnormal indices. Let $m=R(k)+1$, where $R$ is Roseblade's function (Corollary to Theorem 1 of [17]), and choose any $m$ elements $g_{1}, \cdots, g_{m}$ of $G$. Write

$$
H=\left\langle N, g_{1}, \cdots, g_{m}\right\rangle \text {. }
$$

$H / N$ is a finite nilpotent group and can be expressed as the product of its Sylow subgroups $\left\{H_{p} / N: p \in \pi\right\}$. Each of the subgroups $H_{p}$ is subnormal in $G$ and thus inherits the bound $k$ for its subnormal indices. Note also that by the second remark after definition 3.1, $\sigma\left(H_{p}: N\right)=1$, and therefore $H_{p}$, satisfying the conditions of theorem 3.2 , is nilpotent.

Since $H / N$ is finite, we deduce that $H$ is nilpotent, by Fitting's theorem, and its nilpotency class is at most $R(k)$, by Roseblade's result. It follows that

$$
\left[g_{1}, \cdots, g_{m}\right]=1,
$$

showing that $G$ itself is nilpotent of class at most $R(k)$.

It may be of interest to consider whether the conditions of theorem 3.2 can be varied without detriment to the conclusion.

EXAMPLE 1. Let $G$ be the semidirect product of the additive group of rational numbers $Q$ and a 2-cycle $\langle x\rangle$ with action defined by $q^{x}=-q$ for each $q \in Q$. It is not difficult to see that for any positive integer $r, \gamma Q\langle x\rangle^{r}=Q$, and hence that all proper subnormal subgroups of $G$ lie in $Q$. Thus each subnormal subgroup of $G$ is normal, and although the only condition of theorem 3.2 that $G$ fails to satisfy is the " $\sigma$-condition", (for clearly $\sigma(G: Q)=G$ ), $G$ is of course not nilpotent. The " $\sigma$-condition" is therefore essential.

EXAMPLE 2. Let $D$ be an infinite dihedral group, the semidirect product of an infinite cyclic group $C=\langle c\rangle$ and a 2-cycle $B=\langle b\rangle$ with action defined by $\left(c^{r}\right)^{b}=c^{-r}$ for all integers $r$. If, for each positive integer $n$, we denote by $D_{n}$ the subgroup $\left\langle c^{2^{n}}, b\right\rangle$, then each $D_{n}$ is subnormal in $D$ and $D_{n} C=D$. It follows that $\sigma(D: C) \leqq \cap\left\{D_{n}: n \geqq 1\right\}=B$. Since $B$ is self-normalising, $\sigma(D: C)=1, D$ does not have the subnormal intersection property and, although satisfying all other requirements of theorem 3.2, $D$ is not nilpotent. The subnormal intersection property is thus essential in the statement of theorem 3.2.

\section{Soluble minimax groups with the subnormal intersection property}

In this section we make use of theorem 3.2 to prove the following result.

THEOREM 4.1. A soluble minimax group has the subnormal intersection property if and only if it has a bound on its subnormal indices. 
Proof. To prove the non-trivial half of the theorem, let $G$ be a soluble minimax group with the subnormal intersection property. By theorem $A$ of [9], $G$ has a normal radicable abelian subgroup A satisfying the minimal condition on subgroups, such that $G / A$ is a finite extension of a torsion-free nilpotent group. If we prove that $G / A$ has a bound on its subnormal indices, then by lemma 2.1 of [13] so also has $G$. Our result will therefore follow if we can establish:

4.1' Let $G$ be a soluble minimax group with a nilpotent normal subgroup $N$ such that $G / N$ is finite. Then $G$ has the subnormal intersection property if and only if $G$ has a bound on its subnormal indices.

ProOF. The implication in one direction is immediate. Suppose that counterexamples to the converse implication exist, where by a counterexample we mean an ordered pair $(G, N)$ such that $G$ and $N$ satisfy the postulates of $4.1^{\prime}, G$ has the subnormal intersection property but has no bound on its subnormal indices. We begin with a useful observation.

(i) If $(G, N)$ is a counterexample and $T$ is the torsion subgroup of $N$, then $(G / T, N / T)$ is also a counterexample.

To see this, all we need to verify is that $G / T$ can have no bound on its subnormal indices: but this follows easily from lemma 2.1 of [13] and lemma 1 of [12], since $T$, being a periodic soluble minimax group, satisfies the minimal condition on subgroups, and so is (radicable abelian)-by-finite.

Now it is clear that in any counterexample $(G, N), N \neq G$, so that the set of positive integers $\{|G / N|:(G, N)$ is a counterexample $\}$ has a least element $k>1$. For brevity we will term any counterexample $(G, N)$ with $|G / N|=k$ a "minimal" counterexample. We note two important facts.

(ii) If $(G, N)$ is a minimal counterexample then $N$ is the maximal nilpotent normal subgroup of $G$.

This is immediate from our choice of $k$.

(iii) If $(G, N)$ is a minimal counterexample then $\sigma(G: N) \leqq N$ and $(G / \sigma(G: N), N / \sigma(G: N))$ is a minimal counterexample.

To prove (iii), let $H$ be a subnormal subgroup of $G$ with $N \leqq H<G$. It is clear that $(H, N)$ cannot be a counterexample; hence $H$, which of course inherits the subnormal intersection property, must have a bound on its subnormal indices. Moreover since there are only finitely many choices for $H$, we can find a bound $r$ which is independent of the choice of $H$. Now if $S$ is a subnormal subgroup of $G$ such that $S N<G$, it follows that $s(G: S) \leqq s(G: S N)+s(S N: S) \leqq k+r$. Thus the set of non-negative integers $\{s(G: S): S \in \Sigma(G: N)\}$ must be unbounded, and therefore $(G / \sigma(G: N), N \sigma(G: N) / \sigma(G: N))$ must be a counterexample, indeed a minimal counterexample. Hence $|G / N \sigma(G: N)|=|G / N|=k$ and $\sigma(G: N) \leqq N$. We have established (iii).

We now choose a minimal counterexample $\left(G_{1}, N_{1}\right)$ in which $N_{1}$ is torsion- 
free; this is possible by (i). Our aim is to obtain a minimal counterexample $\left(G_{2}, N_{2}\right)$ in which $N_{2}$ is torsion-free and $\sigma\left(G_{2}: N_{2}\right)=1$. If $\sigma\left(G_{1}: N_{1}\right)=1$ we need search no further; if not, we put $M_{1}=\sigma\left(G_{1}: N_{1}\right)$. Then by (iii) $M_{1} \leqq N_{1}$ and $\left(G_{1} / M_{1}, N_{1} / M_{1}\right)$ is a minimal counterexample. If we write $T_{1} / M_{1}$ for the torsion subgroup of $N_{1} / M_{1}$, then $\left(G_{1} / T_{1}, N_{1} / T_{1}\right)$ is also a minimal counterexample, by (i). By repeating this process we construct an ascending chain $M_{1} \leqq T_{1} \leqq M_{2}$ $\leqq T_{2} \leqq \cdots$ of normal subgroups of $G_{1}$, each contained in $N_{1}$, such that for $i \geqq 1$, $\left(G_{1} / M_{i}, N_{1} / M_{i}\right)$ and $\left(G_{1} / T_{i}, N_{1} / T_{i}\right)$ are minimal counterexamples, $T_{i} / M_{i}$ is the torsion subgroup of $N_{1} / M_{i}$, and $M_{i+1} / T_{i}=\sigma\left(G_{1} / T_{i}: N_{1} / T_{i}\right)$.

But now $N_{1}$ is a soluble group of finite reduced rank. Thus by lemma 2.4 there is an integer $n$ such that $M_{n+1} / T_{n}$ is periodic. Since $N_{1} / T_{n}$ is torsion-free this means that $M_{n+1} / T_{n}$ is trivial, that is $\sigma\left(G_{1} / T_{n}, N_{1} / T_{n}\right)=1$. If we now put $G_{2}=G_{1} / T_{n}$ and $N_{2}=N_{1} / T_{n},\left(G_{2}, N_{2}\right)$ is a minimal counterexample, $N_{2}$ is torsion-free and $\sigma\left(G_{2}: N_{2}\right)=1$.

Now $G_{2} / N_{2}$ is a non-trivial finite soluble group; we can therefore find, in $G_{2} / N_{2}$, a non-trivial minimal normal subgroup $Y / N_{2}$ which will be an elementary abelian $p$ group for some prime $p$. Then $Y$ inherits the subnormal intersection property and $\sigma\left(Y: N_{2}\right)=1$ by the second remark after definition 3.1. Thus $Y$ satisfies all the requirements of theorem 3.2, and so is nilpotent. This contradicts (ii) above and shows that in fact no counterexamples exist, completing the proof of the theorem.

\section{A counterexample}

So far in this paper we have shown that under a restriction to soluble minimax groups, the subnormal intersection property is equivalent to the property of having bounded subnormal indices; that is, under this restriction there is equality between the corresponding classes of groups. It would be of interest to know how far this condition can be relaxed before the two classes cease to coincide. In this section we partially answer this question by constructing an example which shows that a restriction to soluble groups of finite reduced rank will not suffice to ensure equality of the two classes.

First we investigate the subnormal structure of a type of finite group which will be used as a building block in a more complex construction.

EXAMPLE 5.1. Let $p$ be an odd prime, and $q$ a prime dividing $p-1$. Let $A$ be a cyclic group of order $p$. Since the automorphism group of $A$ has order $p-1, A$ has an automorphism $\theta$ of order $q$. There is a positive integer $m$ such that for any $a$ in $A, a \theta=a^{m} ; m$ can clearly be chosen so that $1<m<p$. Now for each $a$ in $A$,

$$
a=a \theta^{q}=a^{m^{q}},
$$

from which it follows that $m^{q}-1$ is divisible by $p$. Let $p^{\alpha}$ be the largest power of $p$ dividing $m^{q}-1$. 
Now let $k$ be a positive integer, and $B$ a cyclic group of order $p^{k \alpha}$. Since $m$ is coprime to $p$, the map $x$ defined by $b^{x}=b^{m}$, for each $b$ in $B$, is an automorphism of $B$. We now prove two useful facts about the action of the automorphism group $\langle x\rangle$ on $B$.

(i) Let $B_{1}$ be any subgroup of $B$. If $r$ is a positive integer coprime to $q$ then $\left[B_{1}, x^{r}\right]=B_{1}$.

Proof. Suppose $p \mid m^{r}-1$, that is $m^{r} \equiv 1 \bmod p$. Since $q$ and $r$ are coprime, there exist positive integers $s$ and $t$ such that $s r=1+t q$. Then recalling that $m^{q} \equiv 1 \bmod p$,

$$
m^{s r}=m^{1+t q} \equiv m \bmod p .
$$

But we also have $m^{s r} \equiv 1 \bmod p$, yielding $m \equiv 1 \bmod p$, in contradiction to our choice of $m$. Thus $m^{r}-1$ and $p$ are coprime. If $B_{1}=\left\langle b_{1}\right\rangle$ then

$$
\left[b_{1}, x^{r}\right]=b_{1}^{x^{r-1}}=b_{1}^{m^{r-1}} .
$$

Hence $B_{1}=\left\langle b_{1}\right\rangle=\left\langle b_{1}^{m^{r}-1}\right\rangle \leqq\left[B_{1}, x^{r}\right]$.

To complete the proof of (i) we need only point out that the reverse inclusion is trivial since each subgroup of $B$ is invariant under the action of $\langle x\rangle$.

(ii) Let $B_{1}$ be any subgroup of $B$. Then $\left[B_{1}, x^{q}\right]=B_{1}^{p^{\alpha}}$.

Proof. Let $B_{1}=\left\langle b_{1}\right\rangle$. Then $\left[B_{1}, x^{q}\right]$ is generated by the element

$$
\left[b_{1}, x^{q}\right]=b_{1}^{x^{q}-1}=b_{1}^{m^{q}-1}=b_{1}^{p^{x_{n}}},
$$

where, by the choice of $\alpha, n$ and $p$ are coprime. Thus

$$
B_{1}^{p^{\alpha}}=\left\langle b_{1}^{p^{\alpha}}\right\rangle=\left\langle b_{1}^{p^{\alpha_{n}}}\right\rangle=\left[B_{1}, x^{q}\right]
$$

proving (ii).

It is a simple matter to deduce from (ii) that

$$
B^{\left(x^{q}-1\right)^{k}}=1 \text { but } B^{\left(x^{q}-1\right)^{k-1}} \neq 1 .
$$

If we now consider the semidirect product $G$ of $B$ and $\langle x\rangle$ with the given action, we can describe the subnormal structure of $G$ as follows.

(iv) If a subnormal subgroup $S$ of $G$ contains an element of the form $x^{r} b$ where $r$ is a positive integer coprime to $q$, and $b \in B$, then we can apply (i) above, together with the commutativity of $B$, to deduce that for each non-negative integer $i, B=\gamma B S^{i}$. Thus $B \leqq S$ and $S$ is normal in $G$.

(v) If a subnormal subgroup $S$ of $G$ does not have the property postulated in (iv), then $S$ lies in the subgroup $\left\langle B, x^{q}\right\rangle$ of $G$. By (iii) this subgroup is nilpotent of class precisely $k$, since $B$ is abelian. Thus the defect of $S$ in $G$ cannot exceed $k+1$. 
Indeed (iv) and (v) together imply that $k+1$ is a bound for the subnormal indices in $G$.

We are now in a position to construct the main example of this section.

EXAmple 5.2. We first construct a sequence of pairs of primes. Choose any prime $q(1)$. By Dirichlet's theorem (theorem 15 of [5]) we can find a prime $p(1)$ such that $p(1)-1$ is divisible by $q(1)$.

Suppose now that for $i \geqq 1$ we have chosen pairs of primes $(p(1), q(1)$ ), $\cdots,(p(i), q(i))$ with the following properties:

(a) $q(j)$ divides $p(j)-1$ for $1 \leqq j \leqq i$;

(b) $q(j)<p(j)<q(m)$ if $1 \leqq j<m \leqq i$.

Now choose a prime $q(i+1)>p(i)$ and, as above, apply Dirichlet's theorem to obtain a prime $p(i+1)$ with the property that $q(i+1)$ divides $p(i+1)-1$. In this way we define recursively a sequence $\{(p(i), q(i)): i \geqq 1\}$ of pairs of primes with the properties (a) and (b) for arbitrary $i$.

Now, by the discussion in example 5.1, to each pair $(p(i), q(i))$ there corresponds a positive integer $m(i)$, with $1<m(i)<p(i)$, such that if $A(i)$ denotes a cyclic group of order $p(i)$ then the map $\theta(i)$, defined by $a \theta(i)=a^{m(i)}$ for each $a$ in $A(i)$, is an automorphism of order $q(i)$. Let $p(i)^{\alpha(i)}$ be the largest power of $p(i)$ dividing $m(i)^{q(i)}-1$, and let $B(i)$ be a cyclic group of order $p(i)^{i a(i)}$. Then, as in 5.1 , we can define the action of an infinite cycle $\langle x\rangle$ on $B(i)=\langle b(i)\rangle$ by putting

$$
b(i)^{x}=b(i)^{m(i)}
$$

and extending the definition in the obvious way. The properties of this action and the corresponding semidirect product of $B(i)$ and $\langle x\rangle$ (though now an infinite group) will be precisely as in (i) $-(\mathrm{v})$ of 5.1 , with $k$ replaced by $i$.

If we now denote by $B$ the direct product of the groups $B(i): i \geqq 1$, it is clear that the action of $\langle x\rangle$, defined on each component $B(i)$ as above, can be extended to $B$. Consider the natural semidirect product $G$ of $B$ and $\langle x\rangle$ with this action.

Firstly we note that since $B$ has $p$-rank at most 1 for each prime $p, B$ has reduced rank 1 in the sense of [16] ( $B$ is locally cyclic). Clearly $G$ is then a metabelian group of finite reduced rank, since any finitely generated subgroup can be generated by two elements.

Secondly we show that $G$ has the subnormal intersection property. Consider the standard series of an arbitrary subgroup $S$ of $G$. If $S$ lies in $B$ this series terminates after at most two steps. On the other hand, if $S B>B$ then $S$ must contain an element of the form $x^{t} b$, where $b \in B$ and $t$ is a positive integer. Denote by $D(t)$ the direct product of those subgroups $B(i)$ for which $q(i)$ does not divide $t$. It is easy to see that $B / D(t)$ is a finite group and $D(t)$ is normal in $G$. It follows by lemma 1 of [12] that $G / D(t)$ has a bound on its subnormal indices. But if $q(i)$ does not divide $t$, then by (i) of 5.1 we have 


$$
[B(i), S] \geqq\left[B(i), x^{t} b\right]=\left[B(i), x^{t}\right]=B(i) .
$$

It follows that every term of the standard series of $S$ contains $B(i)$, and hence $D(t)$ also. The standard series of $S$ in $G$ coincides therefore with that of $S D(t)$, and so, by lemma 2.1 (i), must terminate after finitely many steps. Then, again using lemma 2.1, this establishes that $G$ has the subnormal intersection property since $S$ was arbitrary.

Now suppose $G$ has a bound $y$ for its subnormal indices. Choose an integer $i>R(y)$, where $R$ is Roseblade's function (Corollary to Theorem 1 of [17]). $G$ has a factor group isomorphic to $\langle B(i), x\rangle$. This factor group, $G(i)$ say, in herits the bound $y$ for its subnormal indices. But by (iii) of 5.1, $G(i)$ has a normal subgroup which is nilpotent of class precisely $i$ and cannot, by Roseblade's result, have the bound $y$ for its subnormal indices. This contradiction shows that $G$ has unbounded subnormal indices, and completes our investigation of this example.

REMARKS. (i) In [6] it was shown that abelian-by-finite groups with the subnormal intersection property have bounded subnormal indices. This example shows that for abelian-by-cyclic groups in general the corresponding result fails, a fact which does not emerge from Robinson's results on wreath products ([14]).

(ii) We have not been able to decide whether soluble groups of finite total rank (see [16] for definition) with the subnormal intersection property need have a bound on their subnormal indices. On the one hand, the fact that this class is not closed under homomorphisms makes it difficult to handle; on the other hand a counterexample, at least in the spirit of 5.2 , is not easy to construct.

\section{Acknowledgements}

The material in this paper is part of a $\mathrm{PhD}$ thesis submitted to the Australian National University. I am indebted to my supervisor Dr D. McDougall for his generous help and encouragement. This work was carried out during the tenure of an Australian National University research scholarship.

\section{References}

[1] Reinhold Baer, 'Polyminimaxgruppen', Math. Ann. 175 (1968), 1-43.

[2] S. N. Cernikov, 'Complete groups with an ascending central series', (Russian), Mat. Sb. (NS) 18 (60) (1946), 397-422.

[3] P. Hall, 'A contribution to the theory of groups of prime power order', Proc. London. Math. Soc. (2) 36 (1933), 29-95.

[4] P. Hall, 'Some sufficient conditions for a group to be nilpotent', Illinois J. Math. 2 (1958), $787-801$.

[5] G. H. Hardy and E. M. Wright, An introduction to the theory of numbers, 4th edition (Clarendon Press, Oxford, 1960).

[6] D. J. McCaughan, Subnormal structure in infinite soluble groups (Ph. D. Thesis, Australian National University, June 1972). 
[7] D. J. McCaughan, 'Subnormal strucure in some classes of infinite groups', Bull. Austral. Math. Soc. 8 (1973), 137-150.

[8] D.J. McCaughan and D. McDougall, 'The subnormal structure of metanilpotent groups', Bull. Austral. Math. Soc. 6 (1972), 287-306.

[9] David McDougall, 'Soluble minimax groups with the subnormal intersection property', Math. Z. 114 (1970), 241-244.

[10] D. McDougall, 'The subnormal structure of some classes of soluble groups', J. Austral. Math. Soc. 13 (1972), 365-377.

[11] Derek S. Robinson, 'Joins of subnormal subgroups', Illinois J. Math. 9 (1965), 144-168.

[12] Derek S. Robinson, 'On finitely generated soluble groups', Proc. London Math. Soc. (3) 15 (1965), 508-516.

[13] Derek S. Robinson, ‘On the theory of subnormal subgroups', Math. Z. 89 (1965), 30-51.

[14] Derek J. S. Robinson, 'Wreath products and indices of subnormality', Proc. London. Math. Soc. (3) 17 (1967), 257-270.

[15] Derek J. S. Robinson, 'On soluble minimax groups', Math. Z. 101 (1967), 13-40.

[16] Derek J. S. Robinson, Infinite soluble and nilpotent groups (Queen Mary College Mathematics Notes, London, [1968]).

[17] J: E. Roseblade, 'Groups in which every subgroup is subnormal', J. Algebra 2 (1965), 402-412.

Department of Mathematics

Institute of Advanced Studies

Australian National University

Present Addres:

Department of Mathematics

University of Otago

Dunedin

New Zealand. 